\title{
Cirugía menor en un centro de Atención Primaria rural: 2 años de experiencia
}

P. J. Tarraga lópez, A. Celada Rodríguez ${ }^{1}$, M. Cerdán Oliver², J. Solera

Albero $^{3}$, J. M. Ocaña López 33 , M. A. López Cara ${ }^{4}$

Médicos de Atención Primaria Zona 6 de Albacete.

Profesor Honorario de Cirugía Universidad Autónoma de Madrid.

${ }^{1}$ Casas Ibáñez. ${ }^{2} Z$ ona 4 de Albacete. ${ }^{3}$ Alcaraz. ${ }^{4}$ Tarazona. Albacete

\section{RESUMEN}

Objetivo: describir los resultados derivados de realizar cirugía menor (CM) por el médico de Atención Primaria (AP) y analizar el acuerdo diagnóstico prequirúrgico-anatomopatológico.

Diseño: estudio descriptivo, prospectivo.

Participantes: intervenciones realizadas durante dos años por 2 médicos de AP.

Emplazamiento: Centro de Salud de Casas Ibáñez y Servicio de Anatomía Patológica del Hospital General de Albacete.

Mediciones y resultados principales: se analizan variables descriptivas (edad y sexo, localización, diagnóstico lesión, motivo CM (cirugía menor), tipo de intervención, tiempo de espera y resultados histológicos) de todas las intervenciones. Se compara (acuerdo simple) el diagnóstico previo respecto al anatomopatológico.

Se practicaron 425 intervenciones. Se obtuvieron datos completos en 404 personas (229 hombres y 105 mujeres; edad media 42,36 desviación típica 25,03). El motivo más habitual de CM fue el estético, seguido del miedo a malignidad y dolor. Las técnicas quirúrgicas más frecuentes fueron exéresis quirúrgicas 45,7\% y electrocirugía 19,2\%. Las lesiones tratadas fueron principalmente quiste epidérmico 43 casos, verrugas 77 casos y uñas $46 \mathrm{ca}$ sos. Se remitieron al Servicio de Anatomía Patológica 220 casos y el acuerdo diagnóstico con su informe fue del 66,2\%. El tiempo medio de espera fue de 12 dias.
Minor surgery in a rural Primary Health Care center: 2 year's experience

\section{ABSTRACT}

Objective: to describe the results derived from performing minor surgery (MS) for the phisician of Primare Health Care (PHM) and analyse the presurgical-pathology diagnosis concordance in a rural Health Center.

Design: descriptive prospective study.

Participants: interventions performed during two years for two GP.

Setting: Primare Health Care Casas Ibáñez and Pathology Service of General Hospital Albacete.

Measurements and main results: variable descriptives (sex, age, location, lesion diagnosis, motive of $M S$, sort of intervention, awaiting time and histological results) of all interventions were analysed. It was compared (simple according) previous diagnosis with respect to patholoy diagnosis.

Four hundred and twenty-five interventions were performed. Complete data were achieved in 404 persons (229 men, 56,7\% and 175 women, 43,7\%); mean age of 43,9 years old and standard deviation of 23,9).

The most habitual cause for MS was the aesthetic one, continued for malignancy fear and pain. The thecnical of surgery most frequent used were surgical removal in 45,7\% and electrosurgery in 19,2\%. Lesions treated mainly were epidermal cyst 43 cases, warts 77 cases and nails 46 cases. Two hundred and twenty cases were sent to Pathology Service and the diagnosis concordance with its report was of $66,2 \%$. The standby time average was of 12 days. 
Conclusiones: la realización de CM en AP es factible, asumiendo la correcta formación del médico de AP. Dicha actividad en nuestra unidad tiene bajos tiempos de espera y alta concordancia histopalógica.

Palabras clave: Cirugía. Atención Primaria. Concordancia.
Conclusions: performing minor surgery in Primary Health Care is feasible, accepting the proper training of phisician of $P C$. This activity in our health center has low standby times and high histopathological concordance.

Key words: Surgery. Primary Health Care. Concordance.

\section{INTRODUCCIÓN}

La cirugía menor $(\mathrm{CM})$ incluye aquellos procedimientos que tienen como característica común la aplicación de procedimientos quirúrgicos y otras intervenciones sencillas de corta duración, que se realizan habitualmente en tejidos superficiales o de fácil acceso, que precisando en general de anestesia local, tienen poco riesgo y complicaciones postquirúrgicas escasas.

La competencia en CM enriquece y complementa la actividad (preventiva-diagnóstica-terapéutica) del médico de Atención Primaria (MAP) ${ }^{1-3}$.

Si bien en diversos países, sobre todo en Estados Unidos (EE.UU.) o en el Reino Unido, estas prácticas son habituales en el MAP desde hace muchos años, en nuestro país hasta hace 5 años era una práctica inusual como muestra la encuesta de ámbito nacional en 30 unidades docentes de Medicina Familiar y Comunitaria en la que sólo la mitad realizaba algún tipo de CM que incluía básicamente tratamiento de heridas y drenaje de abscesos, pero sólo un $4-14 \%$ hacía CM programada ${ }^{4-10}$.

Desde hace 5 años en que este tipo de procedimientos se incluyó en la Cartera de Servicios del extinto INSALUD, se comienzan a realizar en los Centros de Salud los procedimientos de CM con cierta habituidad.

No obstante la puesta en marcha de estas actividades en una zona básica de salud no está exenta de polémica sobre todo por la patente desconfianza de ciertos especialistas hospitalarios y gestores sanitarios que consideran que deberían realizarse en un medio hospitalario y con cargo a presupuestos del hospital ${ }^{7-14}$.

Existen múltiples argumentos a favor de la realización de la CM en Atención Primaria (AP). El simple hecho de descargar las consultas quirúrgicas hospitalarias de patologías banales que permita dedicar más tiempo a enfermedades de mayor complejidad técnica que requieren tratamiento exclusivo en el hospital. En diversas publicaciones se ha observado que la CM en AP disminuye las listas de espera de las consultas de cirugía general y dermatología.
Por otro lado está el usuario del Sistema Sanitario, al que se le mejora su accesibilidad tanto a consultas diagnósticas como al tratamiento y, lo que cada vez es más importante, el escaso tiempo de demora entre el diagnóstico y el tratamiento. Así mismo, se ha demostrado que esta actividad es satisfactoria tanto para los pacientes como para los profesionales de AP. Por ello, los Sistemas Sanitarios como los de EE.UU. y Reino Unido ofrecen contratos con remuneración específica cuando los Médicos de Familia (MF) realizan estas intervenciones ${ }^{10-18}$.

En nuestro estudio presentamos los resultados descriptivos de la CM en un Centro de Salud rural con las peculiaridades que estos centros tienen. Se analizan también la concordancia entre el diagnóstico prequirúrgico y el resultado anatomopatológico.

\section{MATERIAL Y MÉTODOS}

\section{Emplazamiento}

El Centro de Salud de Casas Ibáñez está situado en la zona noroeste de Albacete, con una cobertura de mas de 16.000 habitantes, distribuidos en 12 municipios y otros tantos núcleos poblacionales, situados en un radio de 21 kilómetros y encontrándose el centro de salud a 50 kilómetros del centro hospitalario de referencia, Complejo Hospitalario Universitario de Albacete. Casas Ibáñez, la localidad que alberga al Centro de Salud está unida con el hospital por dos autobuses que funcionan únicamente de mañana, no existiendo ningún otro transporte público.

Participaron en el estudio 2 MAP y un auxiliar de enfermería con experiencia previa quirúrgica.

\section{Tipo de estudio}

Planteamos prospectivamente un estudio descriptivo, prospectivo de las intervenciones de CM realizadas por los MAP durante 2 años (de no- 
viembre 1997 a 30 de octubre de 1999). Dichas intervenciones serían derivadas siempre si los MAP no realizaban las intervenciones de CM.

\section{Mediciones y materiales}

Durante dos años (2 de noviembre de 1997 a 31 de octubre de 1999) se intervinieron a todos los pacientes subsidiarios de CM en AP en la sala de cirugía del Centro de Salud de Casas Ibáñez (Albacete).

El soporte documental específico estaba formado por: a) hoja de consentimiento informado debidamente firmada y cumplimentada con todos los datos personales; b) hoja de citación; y c) hoja de intervención, en la que se constataban los antecedente personales y si tenía algún tipo de alergias, el tipo de intervención realizada, si se le había pedido análisis anatomopatológico y el tratamiento a seguir.

El estudio anatomopatológico se realizó en aquellos casos en que estaba indicado.

La infraestructura utilizada incluía: la sala de CM del centro de salud, camilla articulada y elevable, lencería, lampara de cirugía móvil; apósitos, guantes y paños fenestrados estériles y el set quirúrgico de cirugía básica estéril. Se dispuso de formol, relación fluida con el Servicio de Anatomía Patológica del Hospital de referencia, sistema de transporte de muestras (que se utiliza habitualmente para el transporte de las extracciones de sangre y otras muestras biológicas).

Para todas las intervenciones se utilizó un set quirúrgico formado por el siguiente material fungible: gasas estériles, esparadrapo, suturas de seda, paño de campo, guantes estériles, mascarillas desechables, formaldehído, solución yodada, jeringas de $5 \mathrm{ml}$, anestesia local $10 \mathrm{ml}$ (bupivacaína) y, cuando fue preciso llevar a cabo estudio anatomopatológico, se utilizó un recipiente de 25 $\mathrm{ml}$ para el envío de muestras al hospital de referencia.

El material quirúrgico no fungible estaba compuesto por: pinza de Adson, tijeras Metzembaum, pinza de Kocher, mosquitos curvos, portaagujas medianos y bisturí con mango.

\section{Análisis estadístico}

Las variables categóricas se describieron como porcentajes con intervalos de confianza (IC) del $95 \%$. Las variables cuantitativas se describieron mediante: media, mediana, moda, rango y desviación estándar (DE):

El paquete estadístico utilizado fue el Epi-Info V.7.01

\section{RESULTADOS}

Durante los dos años del estudio se realizaron 425 intervenciones (234 en el primero y 191 en el segundo). Obteniéndose datos completos en 405 personas (234 en el primero y 171 en el segundo).

Distribución por sexo: el 56,7\% fueron hombres.

Edad: la edad media fue de 42,36 años con una desviación estándar $\pm 25,03$. Los pacientes tenían una edad entre 5 y 90 años.

Motivo de la consulta: el motivo estético fue el más frecuente con un $51 \%$ (IC $38-42 \%$ ), a continuación dolor local $21,2 \%$ (IC:16-29\%) y el miedo a la malignidad con un 19,7\% (IC: 19-29\%).

Localización de la lesión: la principal localización fue la espalda, seguida de cabeza, tórax y cuello.

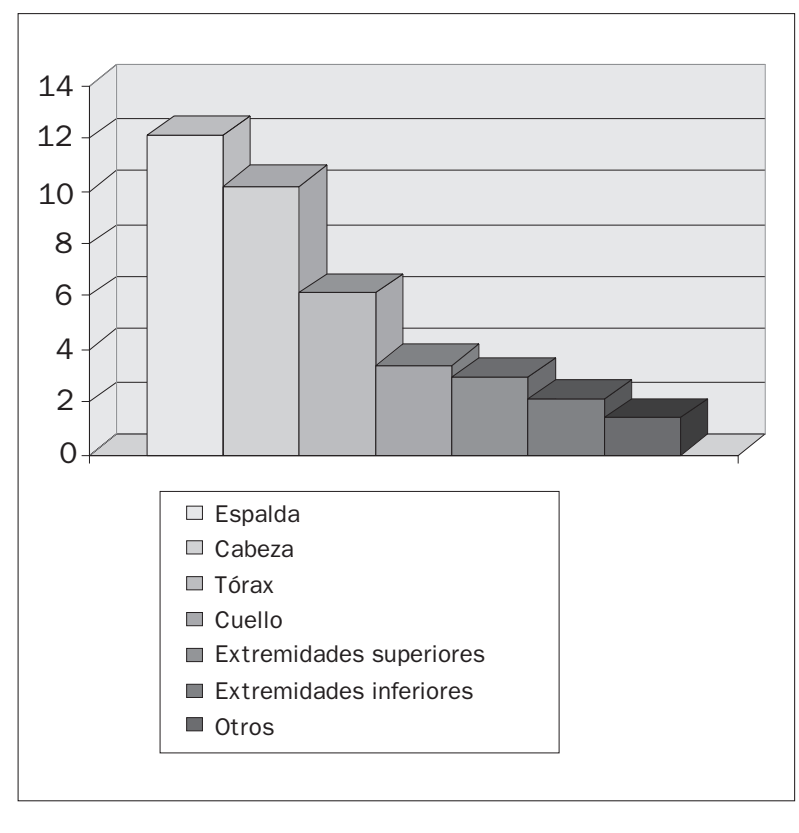

Figura 1

Principales localizaciones de las lesiones.

Tipos de intervenciones: electrocirugía, escisión y cirugía de la uña fueron los principales procedimientos utilizados. En la figura 2 se observa la distribución de las técnicas utilizadas.

Tiempo de espera: la media entre los pacientes tratados en el centro de salud fue de 12 días con una $\mathrm{DE} 15,4$ y un intervalo de 0 días para reparación de heridas y 28 días para lipomas y quistes epidérmicos.

Diagnósticos prequirúrgicos: se hacían constar en la historia clínica y en el informe de la intervención, se relacionan en la tabla I. 




Figura 2

Principales procedimientos utilizados.

Correlación anatomopatológica: se enviaron al laboratorio de anatomía patológica para su análisis 220 lesiones. El acuerdo diagnóstico simple fue del $66,2 \%$ (Tabla II).

\section{DISCUSIÓN}

El claro aumento de la actividad de cirugía menor en los equipos de Atención Primaria ha permitido aliviar la presión de los hospitales, pudiéndose centrar estos en actividades más complejas y especificas.

Otra ventaja que supone el desarrollo de la cirugía menor en Atención Primaria es el aumentar la
Tabla I

\begin{tabular}{|lrl|}
\multicolumn{1}{c}{$\begin{array}{c}\text { PRINCIPALES DIAGNÓSTICOS PREQUIRÚRGICOS Y Y } \\
\text { CONCORDANCIA CON ANATOMÍA PATOLÓGICA }\end{array}$} \\
\cline { 1 - 2 } Diagnósticos & Frecuencia & $\begin{array}{c}\text { Concordancia con } \\
\text { anatomopatológica }\end{array}$ \\
\hline Cuerpos extraños & 2 & \\
Heridas cutáneas & 112 & \\
Abscesos & 8 & $7(25,93 \%)$ \\
Nevus melanocítico & 8 & $5(45,45 \%)$ \\
Queratosis actínica & 3 & $1(100 \%)$ \\
Molusco contagioso & 1 & $18(64,30 \%)$ \\
Queratosis seborreica & 27 & $40(85,11 \%)$ \\
Quiste epidérmico & 43 & $5(58,33 \%)$ \\
Lipomas & 7 & $35(43,75 \%)$ \\
Verrugas & 77 & $35(59,32 \%)$ \\
Patología uñas & 46 & \\
Fibroma & 50 & \\
Otras & 21 & \\
\hline
\end{tabular}

capacidad resolutiva de este nivel sanitario, así como favorece el desarrollo profesional del personal de Atención Primaria ${ }^{1-12}$.

En nuestra experiencia en la Zona Básica de Salud de Casas Ibáñez, los resultados han sido excelentes, tanto cualitativa como cuantitativamente, consolidándose como un servicio más de nuestro centro, que incluso ha llegado a ser casi imprescindible, dándose la anécdota, que durante un tiempo que dejó de darse este servicio, por estar de baja laboral uno de los profesionales médicos que realizaba esta actividad, hubo reclamaciones de los alcaldes de las localidades de la zona a la Dirección Médica de la Gerencia del Área para que se restableciera lo antes posible.

Una ventaja para los usuarios de nuestra zona es que al estar la población muy dispersa y alejada del

Tabla II

\begin{tabular}{|c|c|c|c|}
\hline $\begin{array}{l}\text { Diagnóstico } \\
\text { anatomopatológico }\end{array}$ & Número de casos & $\begin{array}{c}\text { Casos con } \\
\text { diagnóstico correcto }\end{array}$ & Otros diagnósticos \\
\hline Nevus melanocítico & 8 & $7(87,5 \%)$ & $1 \mathrm{vV}$ \\
\hline Queratosis actinica & 3 & $2(66,6 \%)$ & $1 \mathrm{QS}$ \\
\hline Molusco contagioso & 1 & $1(100 \%)$ & \\
\hline Queratosis seborreica & 27 & $18(66,66 \%)$ & $7 \mathrm{NM}-2 \mathrm{QA}$ \\
\hline Quiste epidérmico & 47 & $40(85,10 \%)$ & 5 lipomas- 2 sd \\
\hline Lipomas & 7 & $7(100 \%)$ & \\
\hline Verrugas virales & 77 & $35(45,50 \%)$ & 9 fibromas-10 NM-6 QA-17 sd \\
\hline Fibromas & 50 & $35(70 \%)$ & 3 vv-2 NM-6 QS-4 SD \\
\hline Total & 220 & $145(66,2 \%)$ & \\
\hline
\end{tabular}

$N M=$ nevus melanocitico. $V V=$ verrugas virales. $Q S=$ queratosis seborreica. $Q A=$ queratosis actínica. $S D=$ sin diagnóstico. 
hospital de referencia, estando comunicados sólo por un autobús de ida y otro de vuelta, la realización de estas actividades ha supuesto evitar desplazamientos tanto para consultas como para tratamientos, sobre todo en la población de edad que es la que más frecuente y la que mayores problemas plantea.

Un punto de gran interés es la necesidad de remitir todas las muestras obtenidas tras la cirugía. En nuestro estudio, el MAP manda en su mayoría el producto obtenido a anatomía patológica. Este hecho contrasta con los datos obtenidos en otros estudios en que se enviaban en escasas ocasiones s $^{15-25}$.

La concordancia diagnóstica fue del $66 \%$, cifra alta si comparamos con el 41-62\% que se relaciona en la publicación de MCWilliam et al y aceptable comparada con la de Arribas et al ${ }^{26-29}$.

Estos y otros estudios han mostrado que la CM en AP es más barata para el sistema, mejora la accesibilidad y reduce las listas de espera ${ }^{13,28-33}$.

Es importante en este tipo de prácticas detectar las lesiones potencialmente malignas, para lo cual la formación continuada en interconsultas docentes con el dermatólogo puede ser de gran importancia. Junto con el dermatólogo tomaremos las decisiones más acertadas respecto a la intervención a realizar y la conveniencia de derivación o no de la lesión que ha de tratarse con $\mathrm{CM}^{1,3,33}$.

Las lesiones susceptibles de CM son todas aquellas que están localizadas fuera de las zonas de riesgo (axilas, otras zonas con nervios o tendones,...) en nuestro estudio vemos como la gran mayoría de estas lesiones se encuentran en espalda, tórax, cabeza, donde a pesar de existir mayores problemas cicatrizales secundarios a la técnica sobre todo en las 2 primeras regiones corporales (deshiscencias de sutura) no ha habido secuelas ${ }^{1-3,33}$.

Los motivos más frecuentes de consulta han sido estéticos, dolor local y miedo a la maglinización, probablemente estos hechos son debidos al contacto directo y la relación de confianza existentes entre los pacientes y los MAP.

Unos de los puntos más destacable de nuestro trabajo es el tiempo de espera entre la valoración de la lesión y el tratamiento quirúrgico, que fue de 12 días, siendo de 0 días aquellas lesiones que por su naturaleza eran de relativa urgencia (uñas encarnadas, reparación de heridas,...) y de 28 días para intervenciones de complejidad como extirpación de quistes epidérmicos y lipomas. Consideramos que este tiempo de espera es notablemente más inferior a otros tiempos de espera de cirujanos o dermatólogos en ambulatorios y hospitales ${ }^{33}$.

Hay que considerar también que no ha habido rechazos a la CM en AP y si bien no se ha hecho todavía encuesta de satisfacción podemos reseñar que por métodos indirectos hemos detectado una alta satisfacción ya que venían a demandarnos el servicio familiares y amigos de pacientes intervenidos.

Concluimos pues, que en lesiones susceptibles de CM en AP (diagnóstico morfológico benigno, localizados en territorios corporales sin riesgo) utilizando el instrumental y estructura habitual del centro de salud, con una buena competencia del MAP, la realización de la CM en AP tiene resultados satisfactorios.

\section{CORRESPONDENCIA:}

Pedro J. Tárraga López

C/ Arcángel S. Gabriel, 23 P.4 B.A

02002 Albacete

Telef. 967505263-967241511.-Fax 967520526

e-mail: pedrotar@saludalia.com

\section{Bibliografía}

1. Arribas Blanco JM. Manual de cirugía menor y otros procedimientos en medicina de familia. Madrid: Jarpyo, 2000.

2. Martín Zurro A, Cano Pérez JF. Atención primaria de salud. En: Martín Zurro A, Cano Pérez JF, ed. Atención primaria: conceptos, organización y practica clínica. $4 .^{\text {a }}$ ed. Madrid: Harcourt Brace, 1999. p. 3-16.

3. Arribas JM, Caballero F, eds. Cirugía Menor y otros Procedimientos en la Consulta del Médico de Familia. Madrid: Jarpyo Ed, 1993.

4. Grossman JA. Minor injuries and repairs. Nueva York: Gower Medical, 1993.

5. Menon NK. Minor surgery in general practice. The Practitioner 1986; 230: 917-20.

6. Snell GE. Problemas quirúrgicos. En: Taylor RB, ed. Medicina de Familia, principios y práctica [ed. esp. trad de la $3^{\text {a }}$ de Springer-Verag Inc., 1988]. Barcelona: Doyma, 1991. p. 673-707.

7. Programa Docente de Medicina de Familia y Comunitaria Comisión Nacional de la Especialidad. Consejo Nacional de Especialidades Médicas. Madrid: Ministerio de Salud y Consumo-Ministerio de Educación y Ciencia,1993.

8. Foulkes A. Minor surgery in general practice. $\mathrm{Br}$ Med $\mathbf{J}$ 1993; 307: 685 .

9. Hunt AC, Sherwood A. Competence for GP surgery. Br Med J 1990; 301: 495

10. Arribas Blanco JM. Cirugía Menor y otros Procedimientos en la consulta del Médico de Familia: Situación en España. Ponencia del $14^{\circ}$ Congreso Nacional de Medicina Familiar y Comunitaria. Madrid, 23-26 de noviembre, 1994. 
11. Calnan M. Variations in the Range of Services Provided by General Practitioners. Fam Pract 1988; 5: 94-104.

12. Rodney WM, Richards E, Ounanian LL, Morrison JD. Constraints on the Performance of Minor Surgery by Family Physicians: Study of a "Mock" Skin Biopsy Procedure. Fam Pract 1987; 4: 36-40.

13. Coid DR. General Practitioner Minor Surgery Facilitated by a Fife District General Hospital. Evaluation of a Pilot Project. Health Bulletin 1990; 48/3: 132-6.

14. Brown JS. Should general practitioners perform minor sugery? [editorial]. Br J Hosp Med 1998; 39: 103.

15. Tárraga López PJ, Marín Nieto E, García Olmo D, Celada Rodríguez A, Solera Albero J, Cerdán Oliver M, et al. Impacto económico de la implantación de un programa de cirugía menor en atención primaria. Atención primaria. Vol 27. Num 5. 31 de marzo 2001. p. 335-8.

16. Weddington WH, Gabel LL, Williams T. Surgical practice and perceived training needs of selected ohio family physicians. J Fam Pract 1986; 23: 247-52.

17. Slaler D. Performance of skin biopsies by general practitioners. Br Med J 1991; 305: 1472.

18. Pfenninger JL, Fowler GC. Editores Procedures for Primary Care Physicians. St Louis: Mosby Year Book Inc., 1994.

19. Sodera VK, ed. Minor Surgery In Practice. Cambridge: Cambridge University Press, 1994.

20. O’Cathain, Brazier JE, Milner PC, Fall M. Cost effectiveness of minor surgery in general practice: a prospective comparison with hospital practice. Br J Gen Pract 1992; 42: 13-7.

21. Lowy A, Brazier J, FalI M, Thomas K, Jones N, William BT. Minor surgery by general practitioners under the 1990 contract: effects on hospital workload. Br Med J 1993; 307: 413-7.
22. Hillan KJ, Johnson CP, Morton R. Effects of general practitioner contract on referral of specimens for histological examination. Br Med J 1991; 303: 1080.

23. Corté JA, Peñalver C, Alonso J, Arroyo A. Cirugía menor en Atención Primaria. Aten Primaria 1993; 11: 375-6.

24. McWillian LJ, Knox F, Wilkinson N, Oogarah P. Performance of skin biopsies by general practitioners. Br Med J 1991; 303: 1177-19.

25. Cox NH, Wagstatff R, Poppla AW. Using clinicopathological analysis of general practitioner skin surgery to determine educational requirements and guidelines. Br Med J 1992; 301: 93-6.

26. James AH, Lathman D, Elliott CJ, James RE. Performance of skin biopsies by general practitioners [letter]. Br Med J 1991; 305: 1472-73.

27. Arribas Blanco JM, Martín Martín S, Bru Amantegui S, Villarroel Rodríguez J, Suárez Martín E, Caballero Martínez F. Estudio del acuerdo diagnóstico entre medico de familia y dermatólogo. MEDIFAM 1995; 5: 17-21.

28. Williams RB, Burdge AH, Jones SL. Skin biopsy in general practice. Br Med J 1991; 303: 1179-80.

29. Guereña MJ, Perma C, Gajate J. Correlación clinicopatológica de 370 casos de cirugia menor dermatológica realizada por médicos de familia. Aten Primaria 2001; 28: 320-5.

30. Caro A Minor surgery clinics: How to run one. The Practitioner 1989; 233: 1213-6.

31. James AH, Latham D, Elliott CJ, James RE. Performance of skin biopsies by general practitioner. Br Med J 1991; 305 : 1472-3.

32. Shrank AB. General practitioners and minor surgery. Br J Dermatol 1991; 125: 599-600.

33. Caro A Minor surgery clinics: setting up. The Practitioner 1989; 233: 1136-8. 